\title{
Effects of optical pitch on oculomotor control and the perception of target elevation
}

\author{
MALCOLM M. COHEN \\ NASA-Ames Research Center, Moffett Field, California \\ SHELDON M. EBENHOLTZ \\ State University of New York College of Optometry, New York, New York \\ and \\ BARRY J. LINDER \\ The Permanente Medical Group, Oakland, California
}

\begin{abstract}
In two experiments, we used an ISCAN infrared video system to examine the influence of a pitched visual array on gaze elevation and on judgments of visually perceived eye level. In Experiment 1, subjects attempted to direct their gaze to a relaxed or to a horizontal orientation while they were seated in a room whose walls were pitched at various angles with respect to gravity. Gaze elevation was biased in the direction in which the room was pitched. In Experiment 2, subjects looked into a small box that was pitched at various angles while they attempted simply to direct their gaze alone, or to direct their gaze and place a visual target at their apparent horizon. Both gaze elevation and target settings varied systematically with the pitch orientation of the box. Our results suggest that under these conditions, an optostatic response, of which the subject is unaware, is responsible for the changes in both gaze elevation and judgments of target elevation.
\end{abstract}

According to classical explanations, the visually perceived location of a target in external space depends on the locus of its image on the retina and on concomitant information regarding the direction of the gaze (Hering, 1879/1942; Sherrington, 1918; von Helmholtz, 1866/ 1962). The direction of the gaze, in turn, depends on the orientation of the eyes in the head, and on the orientation of the head relative to an external frame of reference, such as that provided by gravity (Cohen, 1981; Gruesser \& Gruesser-Cornehls, 1986; Matin, 1976; Stoper \& Cohen, 1986), or by surfaces in the external world (Gibson, 1950; Stoper \& Cohen, 1991). Thus, the perceived location of a visual target is changed when any one of the following occurs: (1) The retinal locus of its image is changed but the direction of gaze is maintained; (2) the direction of gaze is changed but the retinal locus of the image remains constant; (3) the magnitude of the change in the direction of gaze does not match the magnitude of the change in the locus of the retinal image; or (4) the interpretation of either the direction of gaze or the locus of the retinal image is changed with respect to the external frame of reference.

This work was supported by NASA Grant UPN 199-16-12-08 to M.M.C. We thank Robert Post, Leland Stone, Arnold Stoper, David Tomko, and Robert Welch for their helpful comments and suggestions on earlier drafts of this manuscript, and Abigail Bautista, Charles De Roshia, Jon Griffith, Dan Gundo, and Thi Huang for their technical support in conducting these studies. Correspondence should be addressed to M. M. Cohen, NASA-Ames Research Center, Mail Stop 239-11, Moffett Field, CA 94035-1000 (e-mail: mmcohen@eos.arc. nasa.gov).
Several investigators have shown that a visual array that is not aligned with gravity can alter the apparent orientations or locations of targets that are viewed against it. One well-known example is the classical rodand-frame phenomenon, whereby a tilted rectangular frame causes a vertical rod seen inside the frame to appear to be tilted in the direction opposite to that of the frame (Asch \& Witkin, 1948; Ebenholtz, 1990). More recently, other investigators have shown that a structured visual array can exert a significant influence on the perceived elevation of a visual target that is viewed against the array when the array is pitched (i.e., rotated up or down about an observer's left-right body axis). The pitched array typically consists of either a small box (e.g., Stoper \& Cohen, 1989) or an entire room (e.g., Matin \& Fox, 1989). However, in a recent series of studies on the specific stimulus components that produce these illusions, Matin and Li $(1992,1994)$ have shown that individual lines that comprise elements of the pitched array yield similar, although somewhat weaker, effects.

These illusions occur not only in the laboratory settings of pitchboxes and pitchrooms, but in natural settings as well, in what have variously been called "magnetic hills," "mystery spots," and "electric braes" (Ross, 1975). The illusions often involve misperceptions of the apparent slope of the terrain and changes in the perceived elevation of objects viewed against the terrain: horizontal surfaces appear to tilt upward or downward, vehicles appear to roll uphill, vertical surfaces appear to be pitched toward or away from the observer, and the ap- 
parent heights of objects and people change as their locations vary with respect to the background.

We believe that this entire class of illusions involving erroneous perceptions of elevation, inclination, and height may be explained by a single putative causenamely, the misjudgment of the elevation of the eyes in the head.

We hypothesize that, without a specific visual target to fixate, the act of viewing a structured visual array elicits an optostatic response (Crone, 1975) - that is, a visually induced change in the orientation of the eyes, of which the observer is unaware. In the context of the current study, this response causes the eyes to rotate with the pitch of the array, toward a line that is normal to the far surface of the array. When the array is pitched upward (i.e., with the top of its far wall tilted toward the observer), the direction of gaze also rotates upward; similarly, when the array is pitched downward (i.e., with the top of its far wall tilted away from the observer), the direction of gaze rotates downward. In both cases, the amount by which the direction of gaze is rotated is less than the amount by which the array is pitched. We suggest that the observer, because he or she is unaware of this change in the direction of gaze, misperceives the location of a target viewed against the array. This vertically acting optostatic response, which we hypothesize to underlie these illusions, has not previously been demonstrated.

On the basis of our hypothesis, we predict systematic changes in the direction of gaze when an individual is exposed to stimulus conditions that cause the illusions. Further, we anticipate that the individual will be unaware that these changes in eye position have occurred. Finally, we predict that, for each individual, the magnitudes of the changes in eye position will be approximately equal to the magnitudes of the illusions that they are proposed to underlie.

In the present experiments, we examined the above conjectures by having one group of subjects view the interior of a pitchroom (Matin \& Fox, 1989), and by having a second group view the interior of a smaller pitchbox (Stoper \& Cohen, 1989), while we recorded the actual position of their eyes in the head with an infrared video camera system (ISCAN). In Experiment 1, we examined eye position both when the subjects were instructed to relax and when they were instructed to align their eyes with the apparent horizon. In Experiment 2, we again examined eye position when the subjects attempted to align their eyes with the apparent horizon, but in addition, in a separate session, we measured both eye position and target position when the subjects attempted to place a visual target at the apparent horizon.

\section{EXPERIMENT 1}

\section{Method}

Subjects. Sixteen individuals (13 males and 3 females) served as subjects in this experiment; they ranged in age from 18 to 55 years, with a median age of 24 years. Of the subjects, 14 were naive as to the purpose of the study and were paid for their services, and 2 ( $\mathrm{S} 1$ and $\mathrm{S} 2$, who were also authors of this paper) were fully aware of the experiment and were not paid to serve as subjects. All subjects had 20/30 vision or better, either without correction or as corrected by eyeglasses or contact lenses.

Materials and Procedure. The subjects wore a helmet-mounted ISCAN infrared video system while comfortably seated upright in an adjustable chair with their eyes at the pivot axis of a pitchroom, as depicted in Figure 1. The pitchroom (see Nemire \& Cohen, 1993) was constructed on an aluminum framework, with its pivot axis at the center of the long side wall; it measured $1.22 \times 2.79 \times$ $1.68 \mathrm{~m}$ (width $\times$ length $\times$ height). The framework was fitted with foam-core walls that were coated on their interior sides with wallpaper, providing a grid-within-a-grid pattern consisting of squares that measured $5.2 \times 5.2 \mathrm{~cm}$, each consisting of 64 smaller squares that measured approximately $6.5 \mathrm{~mm}$ on each side. Dark blue lines, printed on a white background, defined both grids; the lines forming the larger grid were approximately $3 \mathrm{~mm}$ thick, and the lines forming the smaller grid were approximately $0.5 \mathrm{~mm}$ thick. To eliminate unwanted external references, black drop cloth, which also covered the floor of the room, was hung from the bottom of each wall to the floor.

Each subject's head was stabilized with the aid of a custommolded bite board. The subjects were instructed to view the far wall of the pitchroom after the room had been set at each of the following five orientations: (1) pitched down $20^{\circ}$; (2) pitched down $10^{\circ}$; (3) level (far wall vertical); (4) pitched up $10^{\circ}$; and (5) pitched up $20^{\circ}$

Each subject viewed the far wall of the pitchroom under two different sets of instructions - relaxed and horizontal. Under relaxed instructions, the subjects were told to direct their gaze so that their eyes were in a "comfortable and relaxed position" and so that they did not "exert any effort" that would cause their eyes to be pointed away from that relaxed position. Under horizontal instructions, they were told to direct their gaze so that their eyes were pointed "horizontally, or parallel to the earth and perpendicular to gravity." Half of the subjects (i.e., 8) received the relaxed instructions first, and half received the horizontal instructions first. Except for one subject (S2), whose sessions were only $4 \mathrm{~h}$ apart, sessions for all other subjects were separated by at least $24 \mathrm{~h}$.

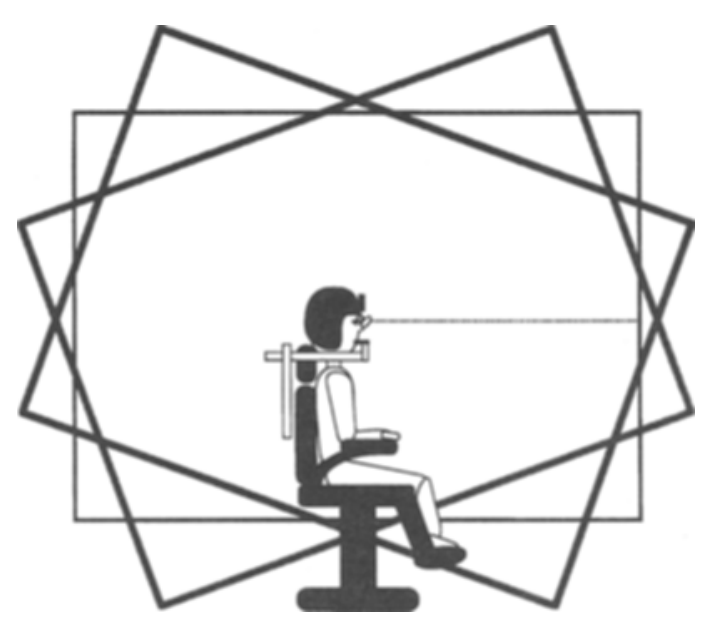

Figure 1. The subject, wearing the ISCAN helmet-mounted video camera, is seated at the center of the pitchroom, which is depicted simultaneously in three orientations: $-20^{\circ}$ (far wall pitched down); $0^{\circ}$ (room level); and $+20^{\circ}$ (far wall pitched up). The subject's direction of gaze is depicted along the true horizontal. 
Half of the subjects (i.e., $8-4$ of whom received relaxed instructions first, and 4 of whom received horizontal instructions first) were asked to direct their eyes according to instructions when the orientation of the pitchroom was changed from $-20^{\circ}$ to $+20^{\circ}$ in $10^{\circ}$ steps, and then from $+20^{\circ}$ to $-20^{\circ}$ in $10^{\circ}$ steps; the remaining 8 subjects ( 4 from each group, as above) were given the reverse sequence of pitchroom orientations. At the start and at the end, as well as for two trials in the middle, of each sequence, the pitchroom was totally darkened. The subjects were instructed to close their eyes immediately before the orientation of the pitchroom was adjusted, and to open them when the adjustment was completed. To prevent the results being affected by possible aftereffects of the immediately preceding pitch condition, we placed the pitchroom in an erect orientation between each setting, and required the subjects to view the far wall of the room for at least $10 \mathrm{sec}$. The experimenter continuously monitored the subjects' eyes with the ISCAN system to ensure that they were following instructions. When the subjects felt that their eyes were in the orientation requested by the experimenter, they were to signal him, and keep their eyes in that orientation for approximately $5 \mathrm{sec}$ longer, during which time the experimenter would record the objective position of their eyes with the ISCAN system.

Calibration. Because we were initially concerned that the subjects would become fatigued during the course of the study, and because we did not want the potential effects of fatigue to influence the results, we conducted the calibrations only after each set of experimental data had been obtained. At the end of each session, the subjects remained seated in the pitchroom while we obtained calibration recordings of eye position during target fixation. The calibrations were used to evaluate changes in eye position that were obtained during the data collection session that immediately preceded them. Each subject was instructed to fixate a target (a black, circular, 6.3-mm-diam dot) on the far wall of the room while the target was set at each of the following five different positions: (1) $20^{\circ}$ above eye level; (2) $10^{\circ}$ above eye level; (3) eye level; (4) $10^{\circ}$ below eye level; and (5) $20^{\circ}$ below eye level. Data were sampled at a rate of $60 \mathrm{~Hz}$, and 5 -sec samples were found to be more than adequate: across all calibrations, measures of eye position versus target position yielded a within-subject $r^{2}$ better than 0.99 , and eye position could be measured to an accuracy of at least $0.5^{\circ}$.

\section{Results and Discussion}

Eye position with pitchroom visible. Mean settings of eye position as a function of the orientation of the pitchroom and the instructions given to the subjects are depicted in Figure 2. Settings of eye position under both relaxed and horizontal instructions changed with the pitch of the room $[F(4,60)=42.62, p<.001]$; they differed from one another according to the specific instructions given $[F(1,15)=8.46, p<.05]$, and the interaction between the pitch of the room and the instructions was also significant $[F(4,60)=4.56, p<.01]$.

As determined by linear regression analysis, the mean position of the eyes changed with a slope of $0.27^{\circ}$ per degree of room pitch $[t(15)=5.23, p<.001]$ under relaxed instructions, and with a slope of $0.37^{\circ}$ per degree of room pitch $[t(15)=8.16, p<.001]$ under horizontal instructions. The $0.10^{\circ}$ difference in slope between the two sets of instructions was also significant $[t(15)=$ $2.64, p<.02]$, consistent with the previous analysis. Similarly, the zero-intercepts differed as a function of instructions; under the relaxed instructions, the mean intercept was $-5^{\circ}[t(15)=3.54, p<.01]$; under horizontal instructions, it was $-1.6^{\circ}[t(15)=1.25, p>.20]$; the

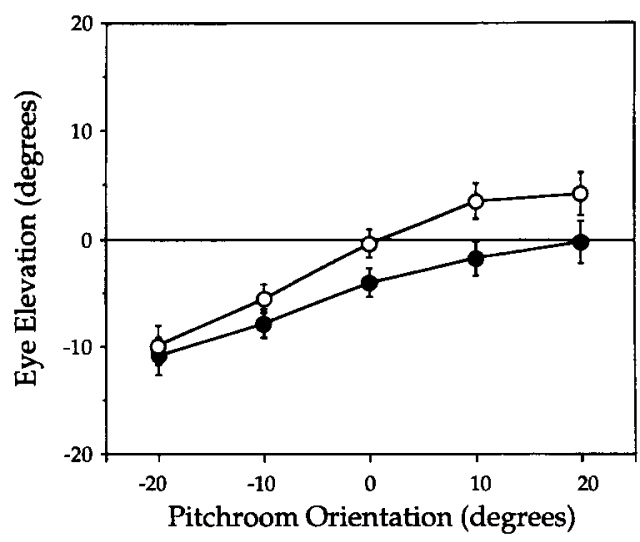

Figure 2. Effects of pitchroom orientation on mean eye elevation under relaxed and horizontal instructions. Solid circles depict mean values for relaxed instructions; open circles depict mean values for horizontal instructions. Error bars represent \pm 1 SEM.

difference of $3.4^{\circ}$ was statistically significant $[t(15)=$ $3.00, p<.01]$.

Figure 3 illustrates, on a subject-by-subject basis, the effect of room pitch on eye position. Although there are exceptions, as well as fairly large individual differences, the general effects depicted in Figure 2 can also be observed in several of the individual subjects.

Collectively, these data show that the static position of the eyes changes with the orientation of the surrounding pitchroom, despite the subjects' attempts to maintain their gaze according to a constant and fixed criterion (i.e., horizontal or relaxed). The results suggest that an optostatic response reduces the angle between the direction of gaze and a line that is normal to the far surface of the room. The influence of the pitchroom is greater when the subjects actively attempt to direct their gaze so that their eyes are pointed toward the horizontal than it is when they simply attempt to relax their eyes in the head.

The differences in settings of eye position under the relaxed versus under the horizontal instructions may be due to differences in task demands that are imposed on the subjects when they attempt to follow the instructions. Whereas the horizontal instructions require the subjects to position their eyes relative to an external spatial frame of reference that depends both on the orientation of the eyes in the head and on the orientation of the head with respect to gravity, the relaxed instructions only require the subjects to position their eyes so that they are comfortable relative to the head. Thus, it is difficult to attribute the changes in eye position under relaxed instructions to a change in the interpretation of the external frame of reference.

Eye position with pitchroom in total darkness. When the pitchroom was totally dark, the subjects directed their gaze $6.02^{\circ}$ below the objective horizontal when they were instructed to relax $[t(15)=4.51, p<$ $.001]$, and $3.59^{\circ}$ below the objective horizontal when they were instructed to place their eyes at horizontal 


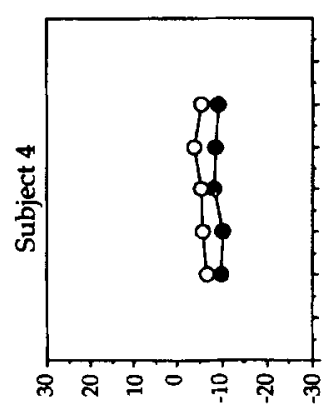

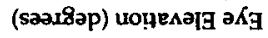
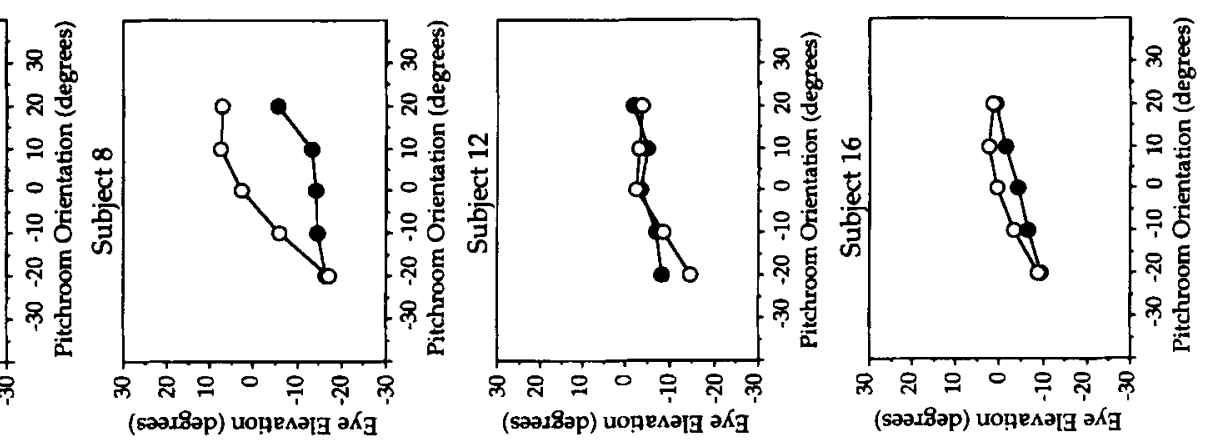

造
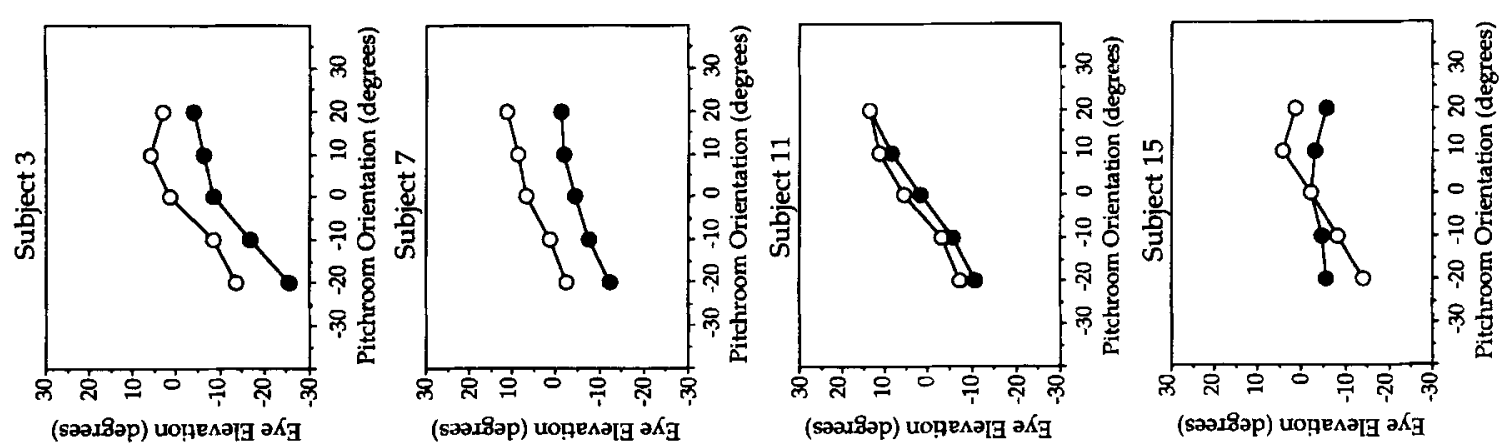

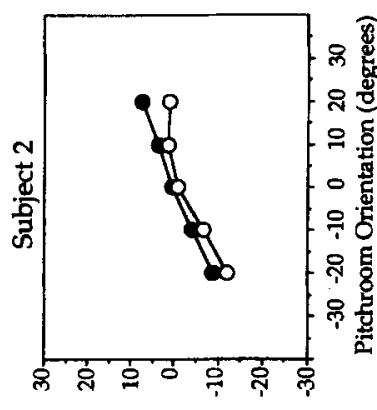

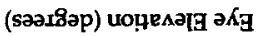

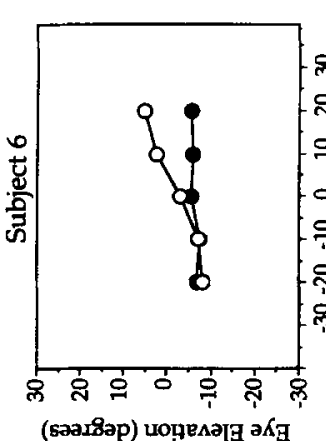

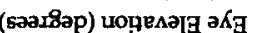
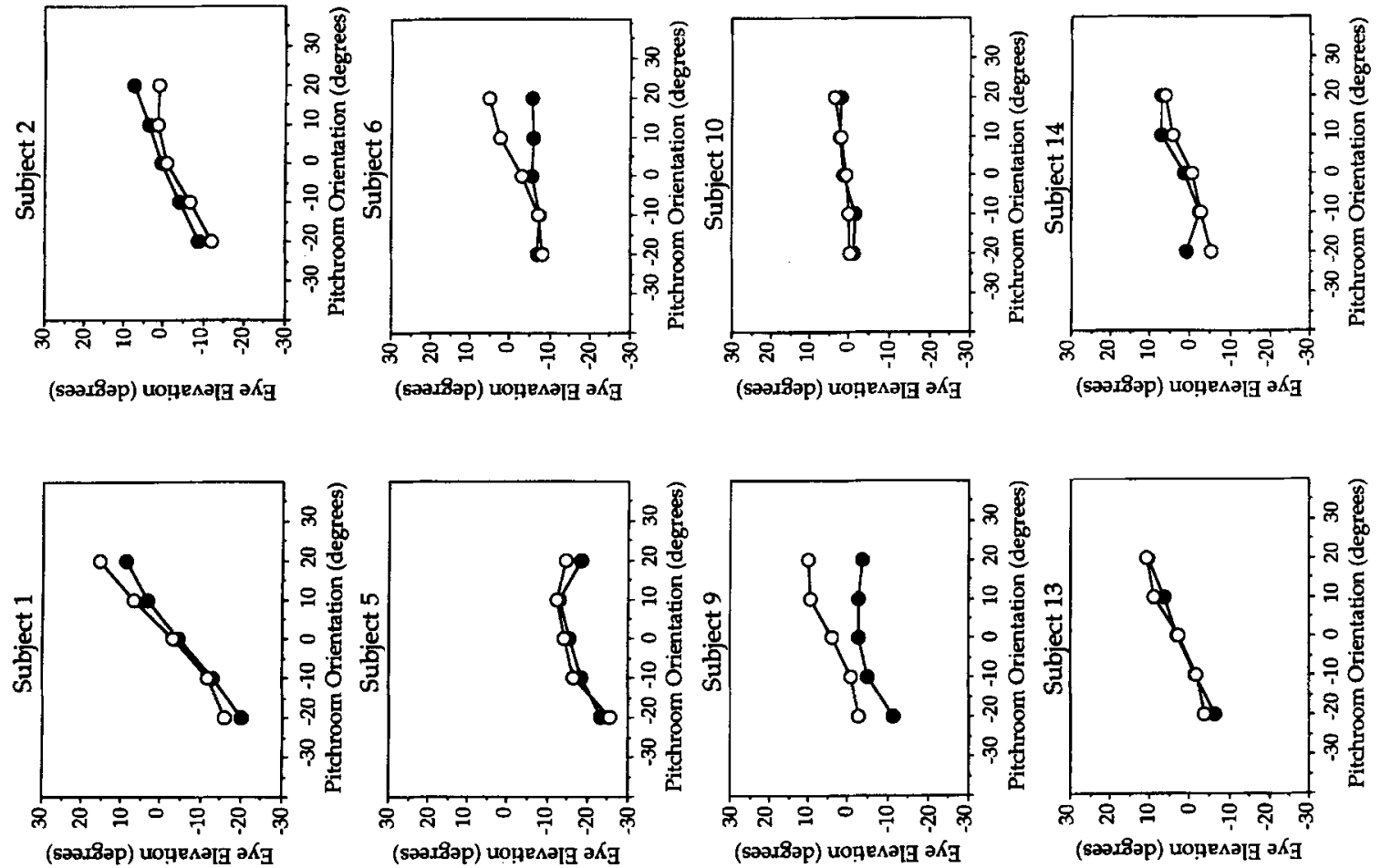
$[t(15)=2.91, p<.02]$. Thus, in the dark, the direction of gaze is significantly below the objective horizontal under both relaxed and horizontal instructions. This finding is consistent with the observations reported by Stoper and Cohen (1986), whereby a single spot of light at the horizon appears to lie above the true horizon when it is viewed against a featureless dark background. The $2.43^{\circ}$ difference in eye position between the relaxed and horizontal instructions was not statistically significant $[t(15)=1.35, p>.20]$ in the dark.

Eye position with pitchroom visible versus eye position with pitchroom in total darkness. We next compared the position of the eyes when the subjects operated under relaxed instructions with that when they operated under horizontal instructions, both when the pitchroom was visible and oriented at $0^{\circ}$ (i.e., no pitch), and when it was in total darkness. Analyses of variance and $t$ tests revealed that both the visibility of the room $[F(1,15)=14.61, p<.01]$ and the specific instructions given $[F(1,15)=5.10, p<.05]$ independently affected the direction of gaze, but that their interaction was not significant. When the room was seen in a level orientation, the mean direction of gaze was $2.22^{\circ}$ below the objective horizontal $[t(15)=2.03, p>.05]$; when the room was darkened, the mean direction of gaze was $4.81^{\circ}$ below the objective horizontal $[t(15)=5.27, p<.001]$. Under relaxed instructions, the direction of gaze was $5.04^{\circ}$ below the objective horizontal $[t(15)=4.19, p<$ $.001]$. Under horizontal instructions, the mean direction of gaze was $1.99^{\circ}$ below the objective horizontal $[t(15)=$ $1.80, p>.05]$. Thus, the subjects tended to place their eyes lower in the dark than in the light (if the surrounding optic array was level), and lower when they were relaxed than when they were attempting to place their eyes in a horizontal orientation.

\section{EXPERIMENT 2}

\section{Method}

Subjects. Sixteen subjects ( 12 male and 4 female) served in this experiment. They ranged in age from 19 to 56 years, with a median age of 21.5 years. Of the subjects, 15 were naive as to the purpose of the study and were paid for their services; one of the subjects (S6, also an author of this paper) was fully aware of the experiment and was not paid to serve as a subject. All of the subjects had 20/30 vision or better, either without correction or as corrected by contact lenses. Three of the subjects had previously participated in Experiment 1 .

Materials and Procedure. Each subject wore the same helmetmounted ISCAN infrared video system that was used in Experiment 1 , while comfortably seated upright in a chair positioned so as to place the subject's eyes at the same height as the pivot axis of a pitchbox measuring $30.5 \times 30.5 \times 45.7 \mathrm{~cm}$ (depth $\times$ width $\times$ height; see Figure 4). The pivot axis ran horizontally through the center of the far surface of the box, and the distance from the bridge of the subject's nose to the pivot axis on the far wall was $53.5 \mathrm{~cm}$. The interior of the box was fitted with 8 electroluminescent strips that were approximately $6.3 \mathrm{~mm}$ wide and defined all eight interior edges of the box. A light-emitting diode (LED) was mounted in a track that ran from the top to the bottom of the box along the center of the far wall. An electric motor, operable by ei-

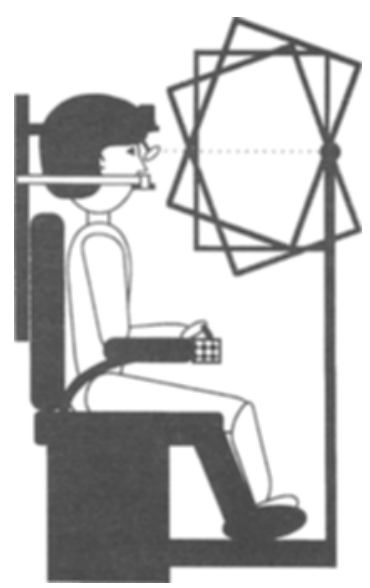

Figure 4. The subject, wearing the ISCAN camera, views a target at eye-level horizontal on the far wall of the pitchbox, which is depicted simultaneously in three orientations: $+20^{\circ}$ (top of far wall rotated toward subject); $0^{\circ}$ (pitchbox level); and $-20^{\circ}$ (top of far wall rotated away from subject). The toggle switch on the armrest allows the subject to adjust the vertical position of the target inside the pitchbox.

ther the subject or the experimenter, was used to adjust the vertical position of the LED in its track. Another motor, operated only by the experimenter, was used to set the pitch orientation of the box.

The subject's head was stabilized with the aid of both the helmet and a custom-molded bite board, which were rigidly attached to the chair. The subject was instructed to view the far wall of the pitchbox while the box was set at each of the following five different orientations: (1) pitched down $20^{\circ}$; (2) pitched down $10^{\circ}$; (3) level (far wall vertical); (4) pitched up $10^{\circ}$; and (5) pitched up $20^{\circ}$.

Each subject viewed the far wall of the pitchbox, and performed each of two tasks in separate sessions: In Task A, with no visible target, the subject attempted to adjust his or her direction of gaze so as to be horizontal (i.e., perpendicular to gravity and parallel to the surface of the earth); in Task B, with the target illuminated, the subject attempted to adjust his or her direction of gaze so as to be horizontal, and simultaneously attempted to set the position of the target so that it appeared to be at his or her horizon. For each subject, individual sessions were separated by at least $24 \mathrm{~h}$.

Of the 16 subjects, 8 were asked to perform according to instructions when the orientation of the pitchbox was changed from $-20^{\circ}$ to $+20^{\circ}$ in $10^{\circ}$ steps, and then from $+20^{\circ}$ to $-20^{\circ}$ in $10^{\circ}$ steps. The remaining 8 subjects were given the reverse sequence of pitchbox orientations. For each sequence, 4 subjects were given Task A first, and 4 were given Task B first. The subjects closed their eyes whenever the orientation of the pitchbox was changed, and opened them when instructed to do so. The experimenter continuously monitored the subjects' eyes with the ISCAN system to ensure that they were following instructions. For Task A, when the subjects felt that their direction of gaze was horizontal, they were to inform the experimenter, and keep their eyes fixed for approximately $2 \mathrm{sec}$ longer, during which time the experimenter recorded the objective position of their eyes with the ISCAN system. For Task B, when the subjects felt that both the target and their direction of gaze were at the horizon, they were to inform the experimenter, and keep their eyes fixed for approximately $2 \mathrm{sec}$ longer, while the experimenter recorded both the objective position of their eyes with the ISCAN system and the objective position of the target on the far wall of the box with a digital computer using a Labtech Notebook program.

Calibration. Our initial concern that the subjects would become fatigued during the course of the study was unfounded; for 
this second experiment, we conducted the calibrations both before and after each set of experimental data had been obtained. The means of the pre- and post-run calibrations were used to evaluate changes in eye position that were obtained during the intervening data-collection session. Each subject was instructed to fixate the target LED on the far wall of the pitchbox while it was set at each of the following five different positions: (1) $18^{\circ}$ above eye level; (2) $10^{\circ}$ above eye level; (3) eye level; (4) $10^{\circ}$ below eye level; and (5) $18^{\circ}$ below eye level. It was found that $2-\mathrm{sec}$ samples of eyeposition data were more than adequate to specify the position of the eyes; in fact, fewer eye-blink artifacts were obtained in this study than in the first experiment, and the 2-sec samples of data, at a rate of $60 \mathrm{~Hz}$, were extremely stable. As in the previous experiment, calibration measures of eye position versus target position yielded a within-subject $r^{2}$ better than 0.99 ; also, we again found that eye position could be specified with an accuracy of at least $0.5^{\circ}$.

\section{Results and Discussion}

Measures of eye position and target position. Mean settings of eye position and target position, as a function of the orientation of the pitchbox and the elevation, are illustrated in Figure 5. Settings of eye position both with the target and without the target, and of target position all changed significantly with changes in the orientation of the pitchbox $[F(4,60)=109.52, p<.001]$; these three different measures of the apparent horizontal did not significantly differ from one another $[F(2,30)=1.01, p>.05]$.

As determined by linear regression analysis, the mean rate at which settings of the eye and of the target change with the orientation of the pitchbox was $0.34^{\circ}$ per degree of box pitch $[t(15)=11.52, p<.001]$. The zero-intercepts did not differ significantly either from zero $[t(15)=1.97$, $p>.05]$ or from one another $[F(2,15)=1.01, p>.05]$.

Figure 6 illustrates, on a subject-by-subject basis, the effect of box pitch on eye position and on settings of the target to the apparent horizon. Although individual differences are clearly present, the general effects that were depicted in Figure 5 can also easily be observed for most individual subjects.

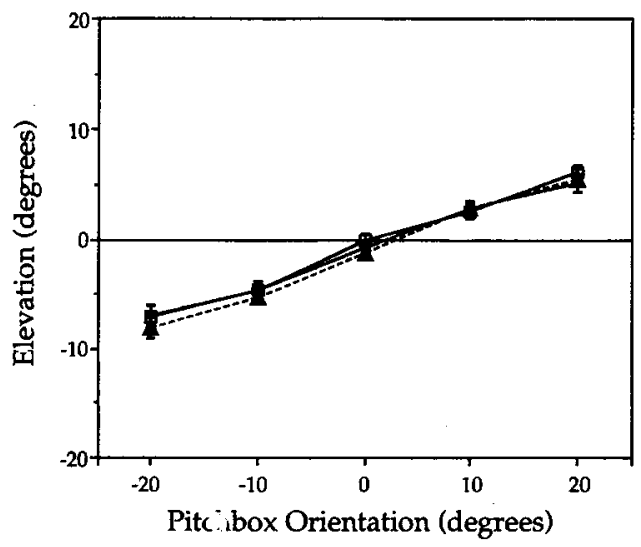

Figure 5. Effects of pitchbox orientation on settings of target and eye elevation to the apparent horizon when the pitchbox is oriented at $-20^{\circ},-10^{\circ}, 0^{\circ},+10^{\circ}$, and $+20^{\circ}$. Triangles represent mean target settings; circles represent mean settings of the eyes in the presence of the target; squares represent mean settings of the eyes alone, when no target is presented. Error bars represent \pm 1 SEM.
Figures 7 and 8 reveal the very strong relationship between target elevation and eye elevation that was obtained in Experiment 2. As illustrated in Figure 7, the regression analysis of the 80 paired values of target and eye elevation, when both measures were obtained in the same session, yields a slope of 1.05 with a standard error of 0.04 , an intercept of -0.31 with a standard error of 1.79 , and an $r^{2}$ of .91 . Even when target settings from one session are compared with measures of eye elevation obtained in a separate session, as shown in Figure 8, the regression yields a slope of 0.87 with a standard error of 0.06 , an intercept of -0.68 with a standard error of 2.95 , and an $r^{2}$ of .75 .

Taken together, these data show that the static position of the eyes, when subjects attempt to set them to a constant and fixed position, changes with the orientation of the pitchbox. Settings of a visual target to the apparent horizon also change with the orientation of the pitchbox. The changes in the settings of the eyes are virtually identical in magnitude to the changes in the settings of the target, suggesting that subjects mislocalize settings of the target because they misjudge the position of their eyes in the head. Our results strongly suggest that an optostatic response, which reduces the angle between the direction of gaze and a line that is normal to the far surface of the box, is responsible for the illusory changes in apparent target elevation that we found in this study.

\section{CONCLUSION}

Collectively, these experiments demonstrate changes both in resting eye position and in the apparent elevation of a visual target that depend on the orientation of the background visual array. The classical notion that the perceived direction of the gaze is determined exclusively by the objective orientation of the eyes in the head and the objective locus of the retinal stimulus does not take sufficient account either of the structure or of the orientation of the visual array.

When the background visual array is pitched and subjects are instructed either to look toward the apparent horizon or simply to relax their gaze, they tend to realign their eyes so that their direction of gaze is shifted to be more normal with the far surface of the array, although they are unaware of making any such changes. Thus, when the subjects' eyes are positioned according to our instructions, the image of a target at eye level that is presented against a pitched visual array will initially fall at a point that is above or below the fovea, depending on the direction in which the background array is pitched. Because the observer is unaware of this change in the direction of gaze, the target is mislocalized; we believe that the observer mislocalizes the target under these circumstances simply because he or she misjudges the position of the eyes with respect to the head.

In order to fixate a target that is viewed against a pitched array and place its image on the fovea, the subjects would have to issue an efferent command that would overcome the unregistered deviation of the eyes. This altered ef- 

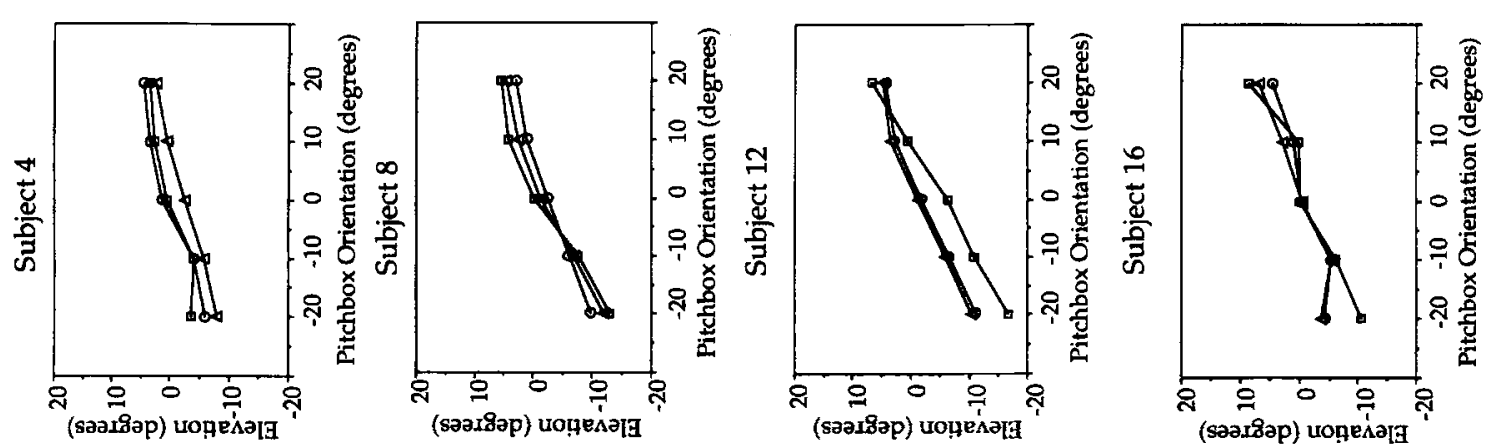

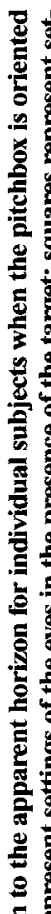
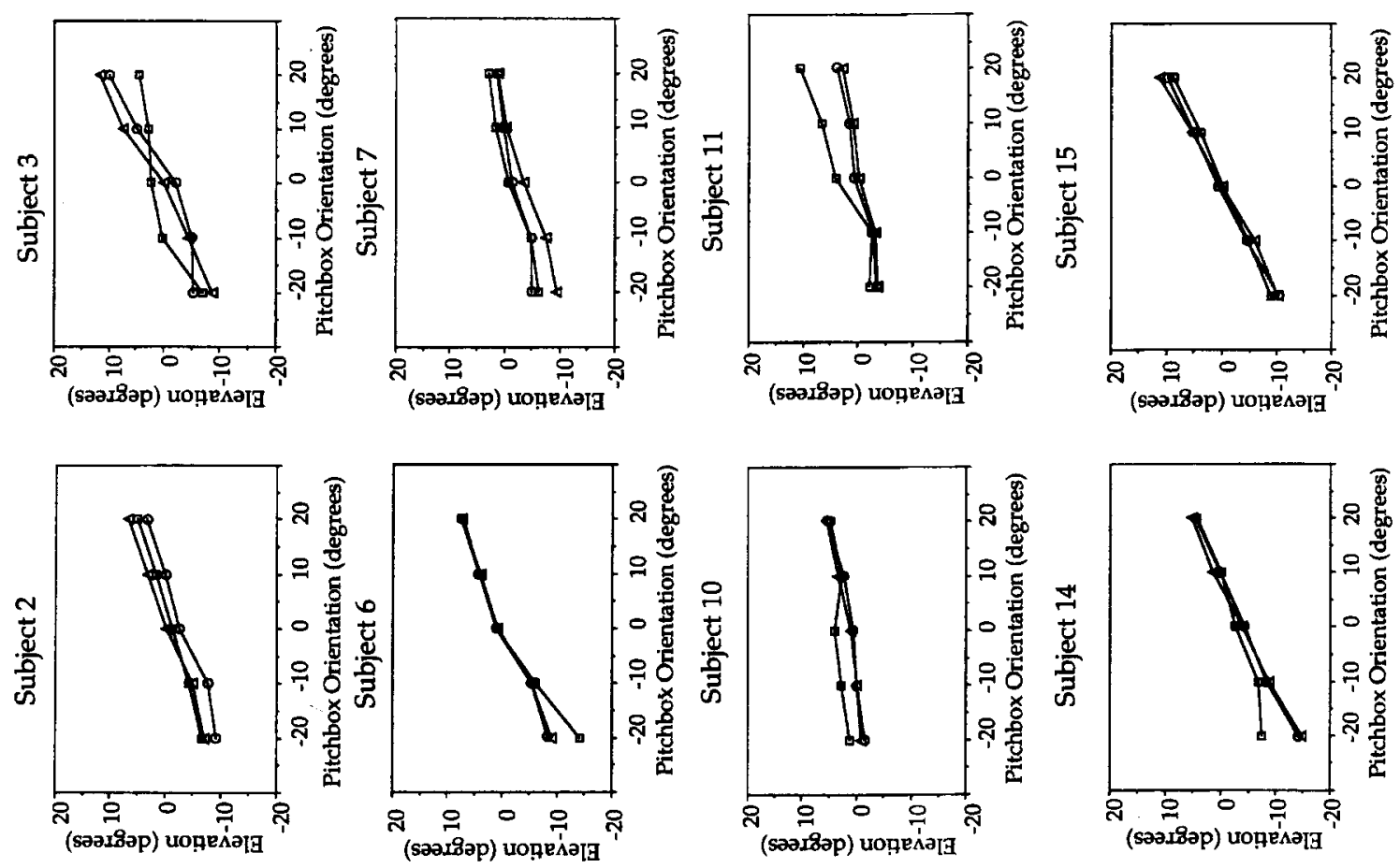

要
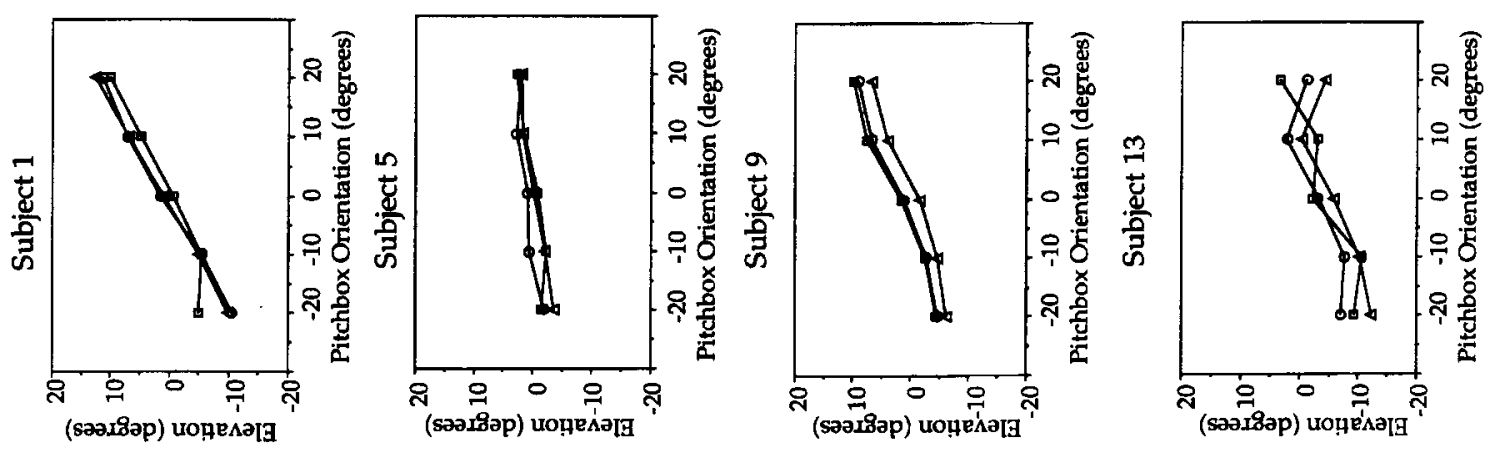

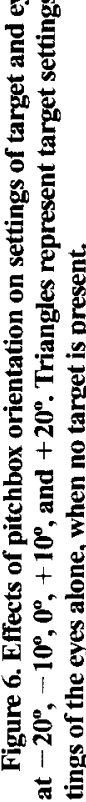




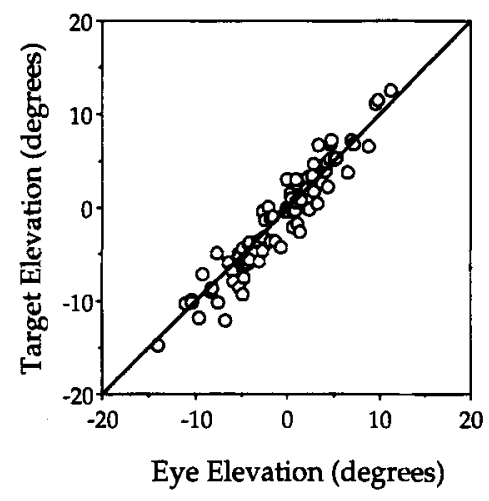

Figure 7. Target elevation as a function of eye elevation (same session). These data consist of 80 paired settings of target and eye elevations across the five pitchbox orientations and 16 subjects who participated in Experiment 2.

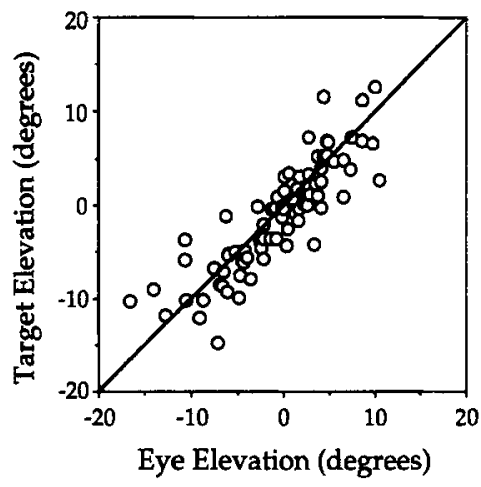

Figure 8. Target elevation as a function of eye elevation (separate sessions). These data were generated by pairing all 80 settings of target elevations that are shown in Figure 7 with measures of eye elevations obtained from the same subjects in separate sessions under identical pitchbox orientations, when no target was presented.

ference, required to direct the eyes for a foveal image, is registered, and it, too, would lead to a mislocalization of the perceived elevation (or height) of the target object.

Thus, whether the target is fixated or not, and whether inflow or outflow is responsible for the error in localizing the target, the relationship between perceived eye position and perceived target position remains invariant.

\section{REFERENCES}

AsCh, S. E., \& WitKIN, H. A. (1948). Studies in space orientation: I. Perception of the upright with displaced visual fields. Journal of Experimental Psychology, 38, 325-327.

COHEN, M. M. (1981). Visual-proprioceptive interactions. In R. D. Walk \& H. L. Pick (Eds.), Intersensory perception and sensory integration (pp. 175-215). New York: Plenum.

Crone, R. A. (1975). Optically induced eye torsion: Il. Optostatic and optokinetic cycloversion. Albrecht Von Graefe's Archiv für Klinische und Experimentelle Ophthalmologie, 196, 1-7.

EBENHOLTZ, S. M. (1990). Metamorphosis from rod and frame to visualvestibular interaction. In I. Rock (Ed.), The legacy of Solomon Asch: Essays in cognition and social psychology (pp. 233-242). Hillsdale, NJ: Erlbaum.

GiBson, J. J. (1950). The perception of the visual world. Boston: Houghton Mifflin.

Gruesser, O.-J., \& GRueSSER-CoRnehls, U. (1986). Physiology of vision. In R. F. Schmidt (Ed.), Fundamentals of sensory physiology (3rd ed., pp. 144-198). New York: Springer-Verlag.

HERING, E. (1942). Spatial sense and movements of the eye (A. Radde, Trans.). Baltimore: The American Academy of Optometry. (Original work published 1879 )

Matin, L. (1976). A possible hybrid mechanism for modification of visual direction associated with eye movements - the paralyzed-eye experiment reconsidered. Perception, 5, 233-239.

Matin, L., \& Fox, C. R. (1989). Visually perceived eye level and perceived elevation of objects: Linearly additive influences from visual field pitch and from gravity. Vision Research, 29, 315-324.

MATIN, L., \& LI, W. (1992). Visually perceived eye level: Changes induced by a pitched-from-vertical 2-line visual field. Journal of Experimental Psychology: Human Perception \& Performance, 18, 257-289.

Matin, L., \& LI, W. (1994). The influence of a stationary single line in darkness on the visual perception of eye level. Vision Research, 34, 311-330.

Nemire, K., \& Cohen, M. M. (1993). Visual and somesthetic influences on postural orientation in the median plane. Perception \& Psychophysics, 53, 106-116.

Ross, H. E. (1975). Behavior and perception in strange environments. New York: Basic Books.

SherRington, C. S. (1918). Observations on the sensual role of the proprioceptive nerve-supply of the extrinsic ocular muscles. Brain, 41, 332-343.

Stoper, A. E., \& Cohen, M. M. (1986). Judgments of eye level in light and in darkness. Perception \& Psychophysics, 40, 311-316.

STOPER, A. E., \& COHEN, M. M. (1989). Effect of structured visual environments on apparent eye level. Perception \& Psychophysics, 46, 469-475.

Stoper, A. E., \& COHEN, M. M. (1991). Optical, gravitational and kinesthetic determinants of judged eye level. In S. R. Ellis (Ed.), Pictorial communication in virtual and real environments (pp. 390403). London: Taylor \& Francis.

voN HELmHOLTZ, H. (1962). Helmholtz's treatise on physiological optics (Vol. 3; J. P. C. Southall, Ed. and Trans.). New York: Dover. (Original work published 1866) 